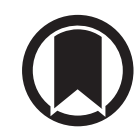

CrossMark

\title{
Early outcomes in MDR-TB and XDR-TB patients treated with delamanid under compassionate use
}

\author{
To the Editor:
}

Multidrug-resistant (MDR) and extensively drug-resistant (XDR) tuberculosis (TB) remain significant global health challenges, with an annual incidence of approximately 480000 and 46000 cases, respectively [1]. Treatment outcomes in MDR-TB patients remain poor overall (52\% success) and outcomes in XDR-TB patients are even more dismal (28\% success) [1-3]. To improve these outcomes, new drugs are needed [4]. Delamanid, a nitroimidazooxazole agent that has been included in the guidelines for MDR-TB treatment by the World Health Organization (WHO), has been shown to improve two-month sputum culture conversion (SCC) and long-term treatment outcomes, when added to an optimised background regimen (OBR) in MDR-TB patients [5-7]. As part of a global access initiative, Otsuka Pharmaceutical Co., Ltd. in coordination with the European Respiratory Society (ERS)/WHO TB Consilium, and Médecins Sans Frontières/Partners in Health (MSF-PIH) established its first Compassionate Use (CU) programme in February 2014 to provide patients with very limited treatment options access to delamanid. We present here early safety and efficacy outcomes of patients enrolled in this effort.

Requests are submitted for initial review to independent committees of either the ERS/WHO TB Consilium or MSF-PIH (Note: requests can be submitted to the TB Consilium from either Otsuka or directly from treating clinicians) [8]. Upon completion of their review, expert summaries and recommendations are generated. These documents, alongside patient data, are then reviewed by the Otsuka CU committee, leading to a decision regarding inclusion in the $\mathrm{CU}$ programme. The requestors are contacted directly by the Otsuka CU committee if additional data are required.

Candidate patients must meet the following inclusion criteria: signed informed consent; pulmonary MDR-TB with limited therapeutic options; unable to participate in a delamanid clinical trial; age $\geqslant 6$ years and weight $>20 \mathrm{~kg}$; and, ability to receive treatment with a background regimen consistent with the WHO guidelines at a treatment centre experienced in MDR-TB management. Exclusion criteria are: prior exposure or intolerance to delamanid; isolated extrapulmonary disease; albumin $<2.8 \mathrm{mg} \cdot \mathrm{dL}^{-1}$; baseline electrolyte imbalances; QT interval corrected for heart rate by the Fridericia's method $>500 \mathrm{~ms}$ (or $>450$ ms with planned concomitant bedaquiline use); $<2$ active or likely to be active drugs (excluding delamanid) to be included in the proposed OBR based on recent drug susceptibility testing results and/or treatment history; and concurrent use of strong cytochrome P450 3A4 (CYP3A4) inhibitors or other experimental anti-TB agents.

Patients who were accepted for CU were treated with delamanid $100 \mathrm{mg}$ twice daily (or $50 \mathrm{mg}$ twice daily for weight $<35 \mathrm{~kg}$ ) for 24 weeks, in combination with an OBR designed in accordance with WHO guidelines and local practice. The OBR was then continued for the duration of treatment. All other subsequent management decisions, including the length of and changes to the regimen, frequency and duration of follow-up, were made by the treating physicians. Pharmacovigilance (PV) training of treating physicians was performed prior to treatment initiation, and safety data were collected throughout the treatment period. Culture-negative status was defined as having one or more negative cultures within 24 weeks after the initiation of delamanid therapy without subsequent positive cultures within that period.

Baseline data from all cases (e.g. demographic information, baseline culture status, drug susceptibility testing results, disease type, presence of cavities, drug resistance category, history of prior treatment, and

@ERSpublications

Early data from the CU programme show delamanid may be effective in MDR/XDR-TB with few treatment options http://ow.ly/tA6Y30cs2Yg

Cite this article as: Hafkin J, Hittel N, Martin A, et al. Early outcomes in MDR-TB and XDR-TB patients treated with delamanid under compassionate use. Eur Respir J 2017; 50: 1700311 [https://doi.org/10.1183/ 13993003.00311-2017]. 
comorbidities) were prospectively collected, entered in a standardised patient access form and recorded in an Excel spreadsheet (Microsoft, Redmond, WA, USA) for further analyses. All adverse events were prospectively captured and reported to the Otsuka PV team during the course of therapy, and assessed for seriousness, severity, and association (causality) with delamanid. In addition, culture information during the 24-week period, confirmation of the regimen given at baseline, use of delamanid, including temporary interruptions, permanent withdrawals from therapy, and completion of a treatment course were retrospectively collected by Otsuka. Descriptive statistics were performed for all patients who initiated treatment with delamanid. Categorical data were reported as counts and proportions, and continuous data were reported as means and ranges.

\begin{tabular}{|c|c|}
\hline Subjects $\mathrm{n}$ & 78 \\
\hline Age years & 32 (8-59) \\
\hline \multicolumn{2}{|l|}{ Sex } \\
\hline Female & $24(31)$ \\
\hline Male & $54(69)$ \\
\hline Weight kg & $54(24-83)$ \\
\hline \multicolumn{2}{|l|}{ Region } \\
\hline Western Europe & $3(4)$ \\
\hline Eastern Europe & $40(51)$ \\
\hline Asia & $18(23)$ \\
\hline Africa & $17(22)$ \\
\hline \multicolumn{2}{|l|}{ Disease type } \\
\hline Pulmonary disease alone & 76 (97) \\
\hline Pulmonary and extrapulmonary disease & $2(3)$ \\
\hline \multicolumn{2}{|l|}{ Cavitary disease } \\
\hline None or unknown & $42(54)$ \\
\hline Yes & $36(46)$ \\
\hline \multicolumn{2}{|l|}{ Resistance type } \\
\hline MDR-TB & $8(10)$ \\
\hline Pre-XDR-TB & $26(33)$ \\
\hline XDR-TB & $44(56)$ \\
\hline \multicolumn{2}{|l|}{ History of prior TB treatment } \\
\hline No & $14(18)$ \\
\hline Yes & $64(82)$ \\
\hline \multicolumn{2}{|l|}{ Comorbidities } \\
\hline HIV co-infection & $12(15)$ \\
\hline HCV co-infection & $11(14)$ \\
\hline HBV co-infection & $3(4)$ \\
\hline Diabetes mellitus & $6(8)$ \\
\hline \multicolumn{2}{|l|}{ Baseline culture status } \\
\hline Negative & $13(17)$ \\
\hline Positive & $65(83)$ \\
\hline \multicolumn{2}{|l|}{ Culture status at 24 weeks $\#$} \\
\hline Negative & $53(80)$ \\
\hline Positive & $11(17)$ \\
\hline Contaminated & $1(2)$ \\
\hline Pending & $1(2)$ \\
\hline \multicolumn{2}{|c|}{ Drugs used in optimised background regimens ${ }^{\#}$} \\
\hline Linezolid & $60(91)$ \\
\hline Clofazimine & $50(76)$ \\
\hline Fluoroquinolones & $45(68)$ \\
\hline Aminoglycosides or capreomycin & $45(68)$ \\
\hline Carbapenems & $33(50)$ \\
\hline Bedaquiline & $12(18)$ \\
\hline \multicolumn{2}{|c|}{ Active drugs in regimens (excluding delamanid) ${ }^{\#}$} \\
\hline Negative culture status at 24 weeks & $3.3(0-5)$ \\
\hline Positive culture status at 24 weeks & $2.3(0-5)$ \\
\hline
\end{tabular}


From February 2014 until June 2016, 112 CU requests were made, 103 approved, six were pending review, one was rejected, and two were withdrawn by the requesting physician. Of those approved, 78 patients had initiated delamanid within the CU programme prior to June 2016. The median time between initial CU request and start of delamanid therapy was 48 days (range 10-449 days). Variability in the time to treatment initiation reflected factors such as regulatory review requirements, shipping time, import and customs delays, and individual factors, such as continued patient interest and/or patient availability. Of the 78 delamanid initiators, 66 completed 24 weeks of treatment with delamanid plus an OBR, four permanently discontinued treatment (one withdrew consent three days later after reconsidering, two were lost to follow-up, and one discontinued treatment because of QT prolongation), and eight died prior to the completion of treatment at a median of 48 days (range 7-174) after starting treatment with delamanid.

Demographic and clinical characteristics of the 78 patients who initiated treatment with delamanid plus an OBR are presented in table 1 . In summary, the mean age of patients was 32 years, $69 \%$ (54 out of 78 ) were male, and the mean weight was $54 \mathrm{~kg}$. Among the patients enrolled, 51\% (40 out of 78) originated from Eastern Europe, 22\% (17 out of 78) were from sub-Saharan Africa and 23\% (18 out of 78) were from Asia. Furthermore, $97 \%$ (76 out of 78 ) of the patients had culture-confirmed disease, $97 \%$ (76 out of 78 ) had pulmonary disease alone, 3\% (2 out of 78 ) had both pulmonary and extrapulmonary disease (one with laryngeal disease and the other with lymph node plus cerebral involvement), and $82 \%$ (64 out of 78) had previously failed TB treatment. In addition, $83 \%$ (65 out of 78) of the patients were culture-positive at referral, 56\% (44 out of 78) had XDR-TB and 33\% (26 out of 78) had pre-XDR-TB (i.e. MDR-TB with additional resistance to either a fluoroquinolone or a second-line injectable agent). Moreover, 15\% (12 out of 78) of the patients had concomitant HIV co-infection and received combination anti-retroviral therapy during MDR/XDR-TB treatment. Hepatitis C virus (HCV)-infection, HBV-infection, and diabetes mellitus were each present in $14 \%$ (11 out of 78 ), 4\% (3 out of 78 ), and $8 \%$ (6 out of 78 ) of all patients, respectively.

Of the 66 patients who completed 24 weeks of treatment with delamanid plus an OBR, 80\% (53 out of 66) achieved culture-negative status at 24 weeks, $17 \%$ (11 out of 66) remained culture-positive at 24 weeks, $2 \%$ ( 1 out of 66 ) had contaminated results and $2 \%$ ( 1 out of 66 ) had pending results. Of these 66 patients, 54 had baseline positive culture data (i.e. prior to starting delamanid therapy). Among that number, $80 \%$ (43 out of 54) had a culture-negative status at 24 weeks, and $20 \%$ (11 out of 54) remained culture-positive. Patients receiving concomitant linezolid, clofazimine, late generation fluoroquinolones (i.e. moxifloxacin or levofloxacin), injectable aminoglycosides or capreomycin, carbapenems, or bedaquiline totalled $91 \%$ (60 out of 66 ), $76 \%$ (50 out of 66 ), 68\% (45 out of 66 ), 68\% (45 out of 66 ), 50\% (33 out of 66), and 18\% (12 out of 66), respectively. The mean number of likely active drugs (based on the results of drug susceptibility testing and treatment history) used in a regimen (excluding delamanid) was 3.3 for those with culture-negative status at 24 weeks, and 2.3 for those with culture-positive status at 24 weeks.

Reports of QT prolongation (defined as corrected QT>500 ms) were noted in 3.8\% (3 out of 78) of the patients exposed to any duration of delamanid. All three patients also received clofazimine and one patient received bedaquiline. In addition, two patients had concomitant hypokalaemia. In all three patients, QT prolongation was resolved without further clinical sequelae (at the time of this report). In two patients, delamanid was re-initiated by the treating physician and a 24 -week treatment course was completed.

Among the eight patients who died prior to the completion of the 24-week treatment course with delamanid, the causes of death included the following: pulmonary haemorrhage, cardiogenic shock, advanced pulmonary disease, pneumothorax, meningoencephalitis, decompensated alcoholic cirrhosis, $\mathrm{HCV}+$ decompensated liver disease with hepatic encephalopathy, and one case with an unknown cause of death (autopsy refused). None of these deaths was considered by the treating clinician as likely caused by delamanid, but rather due to underlying disease processes.

This analysis represents the first published cohort of patients treated with delamanid in predominantly resource-limited settings, outside of clinical trials. Although the data remains preliminary, the majority (80\%) of patients in the CU programme who completed 24 weeks of treatment had encouraging outcomes (i.e. negative 24-week sputum culture status). Early SCC tends to be a positive predictor of long-term treatment outcomes, suggesting that patients with a negative 24-week culture status will also have favourable long-term outcomes [9-11]. This is notable, given that the majority of patients in this cohort had XDR-TB (including many with resistance to other second-line TB drugs, in addition to injectables and fluoroquinolones) and extensive prior treatment histories, and a significant proportion had cavitary disease - features that are all associated with poor treatment outcomes [3, 12]. However, favourable outcomes in this population are consistent with data from the clinical development of delamanid in XDR-TB patients [13]. Safety data among all patients initiating treatment with delamanid revealed no concerns that have not been previously identified in the delamanid development programme [14]. 
Although the absence of a control group, small sample size, and lack of long-term outcomes (including relapse data) are important limitations of this analysis, these results are notable, as they represent a diverse geographic pool of patients who generally would not have qualified for enrolment in a clinical trial.

The CU concept remains relatively novel in the field of TB, because of the historical lack of anti-TB drug development [4]. The international community has expressed a desire for CU frameworks in high TB burden settings that would facilitate more rapid treatment access [15]. However, an evidence-based balance must be struck in $\mathrm{CU}$ programmes to ensure that patients are provided with the best opportunity for positive treatment outcomes, while the already documented risk of resistance amplification [16], potential transmission of highly resistant strains, and the unnecessary exposure to an ineffective regimen are all minimised.

Jeffrey Hafkin ${ }^{1}$, Norbert Hittel ${ }^{2}$, Alexandra Martin ${ }^{2}$ and Rajesh Gupta ${ }^{1}$

${ }^{1}$ Otsuka Pharmaceutical Development and Commercialization Inc., Rockville, MD, USA. ${ }^{2}$ Otsuka Novel Products GmbH, Munich, Germany.

Correspondence: Jeffrey Hafkin, Otsuka Novel Products, 2440 Research Blvd, Rockville, MD, USA. E-mail: jeffrey. hafkin@otsuka-us.com

Received: Feb 132017 | Accepted after revision: April 242017

Conflict of interest: Disclosures can be found alongside this article at erj.ersjournals.com

Acknowledgements: We would like to thank Clotilde Picard and Lusine Breitscheidel (Otsuka Novel Products GmbH, Munich, Germany) for their assistance with data collection, as well as Zuzanna Huebschmann (Otsuka Novel Products $\mathrm{GmbH}$ ) for her critical review of the manuscript.

\section{References}

1 World Health Organization. Global tuberculosis report 2016. WHO/HTM/TB/2016.13. Geneva, World Health Organization, 2016.

2 Jacobson KR, Tierney DB, Jeon CY, et al. Treatment outcomes among patients with extensively drug-resistant tuberculosis: systematic review and meta-analysis. Clin Infect Dis 2010; 51: 6-14.

3 Migliori GB, Sotgiu G, Gandhi NR, et al. Drug resistance beyond extensively drug-resistant tuberculosis: individual patient data meta-analysis. Eur Respir J 2013; 42: 169-179.

4 Lienhardt C, Raviglione M, Spigelman M, et al. New drugs for the treatment of tuberculosis: needs, challenges, promise, and prospects for the future. J Infect Dis 2012; 205: Suppl. 2, S241-S249.

5 Gler MT, Skripconoka V, Sanchez-Garavito E, et al. Delamanid for multidrug-resistant pulmonary tuberculosis. N Eng J Med 2012; 366: 2151-2160.

6 Skripconoka V, Danilovits $\mathrm{M}$, Pehme L, et al. Delamanid improves outcomes and reduces mortality in multidrug-resistant tuberculosis. Eur Respir J 2013; 41: 1393-1400.

7 World Health Organization. The use of delamanid in the treatment of multidrug-resistant tuberculosis. Interim policy guidance. WHO/HTM/TB/2014.23. Geneva, World Health Organization, 2014.

8 Esposito S, D'Ambrosio L, Tadolini M, et al. ERS/WHO Tuberculosis Consilium assistance with extensively drug-resistant tuberculosis management in a child: case study of compassionate delamanid use. Eur Respir J 2014; 44: 811-815.

9 Kurbatova EV, Cegielski JP, Lienhardt C, et al. Sputum culture conversion as a prognostic marker for end-of-treatment outcome in patients with multidrug-resistant tuberculosis: a secondary analysis of data from two observational cohort studies. Lancet Respir Med 2015; 3: 201-209.

10 Wells CD, Gupta R, Hittel N, et al. Long-term mortality assessment of multidrug-resistant tuberculosis patients treated with delamanid. Eur Respir J 2015; 45: 1498-1501.

11 Wallis RS, Pai M, Menzies D, et al. Biomarkers and diagnostics for tuberculosis: progress, needs, and translation into practice. Lancet 2010; 375: 1920-1937.

12 Jeon DS, Kim DH, Kang HS, et al. Survival and predictors of outcomes in non-HIV-infected patients with extensively drug-resistant tuberculosis. Int J Tuberc Lung Dis 2009; 13: 594-600.

13 Gupta R, Geiter LJ, Wells CD, et al. Delamanid for extensively drug-resistant tuberculosis. N Engl J Med 2015; 373: 291-292.

14 Gupta R, Wells CD, Hittel N, et al. Delamanid in the treatment of multidrug-resistant tuberculosis. Int J Tuberc Lung Dis 2016; 20: S33-S37.

15 Horsburgh CR Jr, Haxaire-Theeuwes M, Lienhardt C, et al. Compassionate use of and expanded access to new drugs for drug-resistant tuberculosis. Int J Tuberc Lung Dis 2013; 17: 146-152.

16 Bloemberg GV, Keller PM, Stucki D, et al. Acquired resistance to bedaquiline and delamanid in therapy for tuberculosis. N Eng J Med 2015; 373: 1986-1988. 